\title{
Brigitte Brunet, Le théâtre de Boulevard
}

\section{Luigi Luison}

\section{Q OpenEdition}

\section{Journals}

\section{Edizione digitale}

URL: http://journals.openedition.org/studifrancesi/34017

DOI: 10.4000/studifrancesi.34017

ISSN: 2427-5856

\section{Editore}

Rosenberg \& Sellier

\section{Edizione cartacea}

Data di pubblicazione: 1 décembre 2005

Paginazione: 702

ISSN: 0039-2944

\section{Notizia bibliografica digitale}

Luigi Luison, «Brigitte Brunet, Le théâtre de Boulevard», Studi Francesi [Online], 147 (XLX | III) | 2005,

online dal 30 novembre 2015, consultato il 18 avril 2021. URL: http://journals.openedition.org/ studifrancesi/34017 ; DOI: https://doi.org/10.4000/studifrancesi.34017

\section{Questo documento è stato generato automaticamente il 18 avril 2021.}

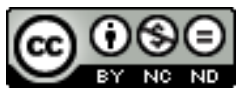

Studi Francesi è distribuita con Licenza Creative Commons Attribuzione - Non commerciale - Non opere derivate 4.0 Internazionale. 


\title{
Brigitte Brunet, Le théâtre de Boulevard
}

\author{
Luigi Luison
}

\section{NOTIZIA}

BRIGITTE BRUNET, Le théâtre de Boulevard, sous la direction de Daniel BERGEZ, Paris, Editions Nathan, 2004, pp. 154.

1 Nel panorama dell'arte drammatica francese, il théâtre de Boulevard appare spesso come un campo poco esplorato. I pochi studi dedicati a questo argomento sono circoscritti, in linea generale, ad un periodo storico, ad un registro o ad una prospettiva particolare, senza tenere conto della sua importanza globale. Brigitte BRUNET, con questo lavoro, si propone di tracciare dei riferimenti precisi in grado di delineare meglio il théâtre de Boulevard nel suo insieme, senza tralasciare un periodo particolare oppure un genere. In quest'ottica il volume è strutturato in tre parti: nella prima, intitolata Théâtre de Boulevard et socété, l'A. descrive dettagliatamente la nascita e i primi anni del théâtre de Boulevard, tracciando la scena teatrale nei secoli XVII e XVIII. Accanto al teatro ufficiale, sovvenzionato dal re, Brigitte Brunet individua gli spettacoli brillanti presentati in occasione delle fiere di Saint-Germain, Saint Laurent e Saint Ovide, il cosiddetto théâtre de la Foire, che è all'origine del théâtre de Boulevard. Les Foires, che occupano un posto di grande rilievo nella storia del teatro parigino, sono luoghi di scambio, gioco, incontro, e toccano un pubblico eteroclito.

2 Nel descrivere le dinamiche di sviluppo del théâtre de Boulevard, l'A. illustra minuziosamente i cambiamenti avvenuti durante il XIX secolo; cambiamenti che consistono in una separazione dei teatri: da una parte i teatri nazionali, nel cuore di Parigi, destinati ai ricchi; dall'altra le sale secondarie, relegate alla periferia, sui Boulevards, destinate ad un pubblico popolare. Situazione che sarà destinata a mutare con l'avvento del prefetto Haussmann (1809-1891), il quale effettuerà una 
ridistribuzione dei luoghi di spettacolo, dovuta essenzialmente ad un cambiamento del pubblico.

3 Nella seconda parte, intitolata Les registres du Boulevard, vengono illustrate le forme di teatro serio praticate sui boulevards. In questa sezione l'A. effettua un'accurata descrizione del melodramma: personaggi, azione, ritmo, elemento patetico, effetti visivi per stupire lo spettatore, fino ad arrivare alla descrizione dei valori espressi dal melodramma stesso: terrore, pietà e valori giudeo-cristiani. In questo modo i théâtres de Boulevard diventano luoghi di uguaglianza sociale in cui il melodramma favorisce la coesione e la comunione sociale. Il genere, uno dei più rappresentati sui boulevards nel corso della prima metà del XIX secolo, sarà poi spodestato dal Vaudeville. A tale proposito l'A. dedica ampio spazio alle caratteristiche fondamentali di coloro che hanno contribuito all'evoluzione del Vaudeville: Eugène Scribe, Eugène Labiche, Georges Feydeau.

4 Nella terza ed ultima parte, intitolata Dramaturgie du Boulevard comique, l'A. espone la struttura del Boulevard comique, spiegando quali sono i livelli di linguaggio ed i registri utilizzati, tenendo conto della complicità fra autori, interpreti e spettatori, che rappresenta proprio la caratteristica fondamentale di questo genere.

5 A conclusione del suo lavoro, Brigitte Brunet tende a chiarire che questo suo studio non vuole contribuire ad alimentare la polemica che vede il popolare théâtre de Boulevard e il cosiddetto teatro intellettuale opposti fra loro. 